\title{
Exposición del modelo DVUC-001 para la realización de productos gráficos en diseño visual
}

\section{Resumen}

Se presenta el resultado de la investigación "Formulación de un método para diseño con base en la simbolización", inscrito en la Vicerrectoría de Investigaciones y Postgrados de la Universidad de Caldas. Los resultados obtenidos ofrecen información relevante sobre la utilización del modelo de método DVUC-001, desarrollado por el "Grupo de Investigaciones Estéticas y Sociales en Diseño Visual", el cual se enfoca en la realización de piezas gráficas dentro de un marco de utilización de tipo académico. El artículo presenta el desarrollo de la investigación y algunos de los resultados más relevantes que se relacionan con la aplicación del modelo.
William Ospina Toro

Magíster en Diseño y Creación Interactiva

Profesor Titular Universidad de Caldas

Manizales, Colombia

Correo electrónico:

william.ospina@ucaldas.edu.co

다이.org/0000-0001-8716-964X

Google Scholar

Recibido: Febrero 2 de 2018

Aprobado: Octubre 15 de 2018

\section{Palabras clave:}

Metodología de diseño, modelo de método, diseño gráfico y

visual. 


\section{Introduction of the DVUC-001 model for making graphic products in visual design}

\begin{abstract}
The result of the research "Formulation of a Method for Design Based on Symbolization", that is registered with code 0748315 in the Research and Graduate Studies Vice-rectory of Universidad de Caldas, is presented. The results obtained offer relevant information on the use of the method model DVUC001 developed by the Aesthetic and Social Research in Visual Design Group, which focuses on the production of graphic pieces within a framework of academic use. The article presents the development of the research and some of the most relevant results that are related to the application of the model.
\end{abstract}

Key words:

Design methodology, method model, graphic and visual design. 


\section{Introducción}

La reflexión sobre los métodos en diseño viene siendo presentada desde hace más de cincuenta años, casi la mitad de la cronología de la actividad académica del diseño; la Escuela de la Bauhaus (1919) está por cumplir cien años de creación y sin embargo en las cinco décadas de estudios y propuestas sobre la metodología del diseño se han logrado pocos acuerdos definitivos. Esto, gracias a los dramáticos cambios que el diseño ha enfrentado en todas sus áreas, a la aparición y desaparición de dispositivos de comunicación, al perfeccionamiento de procedimientos y posibilidades de manipulación de imágenes y objetos, pero sobre todo por el cambio sustancial en las maneras de relación entre los seres humanos.

Uno de esos acuerdos que surgen de asumir al diseño como actividad proyectual es que su ejercicio responde a determinantes de su entorno que hacen del mismo un ejercicio de tipo cultural. Esta hipótesis marca, en gran medida, la concepción o idea que del diseño se tiene en un contexto. Esa relación entre el diseño y el contexto determina la necesidad que intenta resolver el estudio del cual se desprende este artículo, lo que permite deducir que el modelo desarrollado se aleja de la generalidad que caracteriza un método en otra área del conocimiento; particularidad que señala al diseño como una disciplina sui generis como ninguna otra.

De esta manera se presentan las acciones que implicaron el desarrollo y posterior aplicación del modelo DVUC-001 (Diseño Visual Universidad de Caldas) y los aspectos de orden teórico que llevaron al ordenamiento de las fases y estrategias que lo componen. Por otro lado, este ejercicio solo fue posible por la participación decidida de los profesores Gustavo Villa y Walter Castañeda; además de un grupo de estudiantes voluntarios que con sus apreciaciones y trabajo hicieron posible el logro de este modelo. 


\section{Antecedentes}

La utilización de los métodos en diseño al interior de los distintos espacios académicos es una reflexión que se establece en la disciplina del diseño mucho antes de su primera conferencia en 1962 (Rodríguez, 1990); tanto en el área gráfica y más precisamente en el área del diseño industrial, otorgó una importancia relativa a la consideración de una proyectación más racional y menos intuitiva,

después de la segunda guerra mundial dio comienzo en los países industrializados de Europa una gran expansión económica, que la competencia de los países con economía de mercado convirtió en una lucha encarnizada por el mercado internacional. En esta situación el diseño industrial tuvo que adaptarse a las condiciones cambiantes, es decir, no pudo seguir poniendo en práctica métodos creativos subjetivos y emocionales que procedían de la tradición del diseño artístico, en tanto que la industria empezó a racionalizar cada vez más el proyecto, la construcción y la producción. Por ello los diseñadores industriales se esforzaron obviamente en integrar métodos científicos en el proceso proyectual para poder ser aceptados por la industria como interlocutores serios. La Escuela Superior de Diseño de Ulm desempeñó un papel precursor en este aspecto. (Bürdeck, 2002, p. 118)

Desde entonces se han utilizado y adecuado propuestas de método en el diseño, no sin una constante aportación de diversas disciplinas que integran los métodos de manera - al parecer- un poco más clara que la actividad proyectual. Así pues, es latente la necesidad de seguir aclarando la estructura del proceso de diseño con contribuciones aparentemente beneficiosas que han realizado las teorías de la información, de la informática, de la psicología, de la neurología y hasta de la ecología, solo por mencionar algunas.

Sin embargo, estas aportaciones metodológicas traen consigo los marcos de referencia que poseen sus propios campos de acción y reflexión que, al ser trasladados al diseño, limitan el desarrollo mismo de la actividad proyectual como disciplina autónoma. Esta consideración solo significa que reflexionar sobre el método implica pensar y revisar la teoría que lo fundamenta; por ello 
no es posible separarlos o diseccionarlos y únicamente hacer transferencia de uno u otro al campo que nos ocupa.

Ahora bien, esta condición obliga a ser cautelosos a la hora de proponer y presentar un boceto de modelo metodológico para el diseño debido a que las revisiones sobre las regiones de la realidad que las teorías explican son determinantes para delimitar el sentido que se expone en la actividad proyectual y el orden de las estrategias que implican para su explicación. Si teoría y método están relacionados es importante revisar donde surgen las primeras inquietudes de método en diseño para contrastar la teoría que lo fundamenta; de esta manera es posible plantear la existencia de teorías del diseño que sustenten los métodos.

Los factores que impulsaron el desarrollo de métodos en diseño han sido expuestos y se han clasificado desde hace varios años, los tempranos estudios de Luis Rodríguez Morales (1990) permiten ubicar varias causas que determinaron la aparición de métodos en el diseño industrial que en comparación con áreas como el diseño gráfico o la comunicación visual ha tenido un mayor desarrollo; factores de tipo económico y tecnológico componen las determinantes externas al diseño; complejidad del problema, orden pedagógico, orden psicológico y búsqueda de estatus académico, constituyen factores propios del ejercicio proyectual.

De estos últimos, la complejidad del problema merece mayor atención puesto que relaciona de manera directa con la intención de reducir las complicaciones que encierra el proceso de diseño. Esta revisión sobre cómo la complejidad del problema motivó el surgimiento de los métodos en diseño, según Rodríguez (1990), se puede apreciar en la dificultad que caracteriza a la resolución de problemas del diseño relacionada con los contextos en los que se aplica; un entorno con tecnología cambiante deriva en una sociedad en transformación, 
lo que se refleja en necesidades y estilos de vida distintos a los que hasta ese momento se habían identificado y a los que se respondía de manera efectiva mediante, por ejemplo, la producción artesanal. Si la tecnología, la sociedad y la producción han cambiado, la información requerida para dar solución a los problemas que de ellos se desprende es a la vez diversa y creciente. De esta manera se esperaba que el método brindara claridad al proceso de diseño y que además propusiera ciertas directivas sobre el manejo de esos complejos requerimientos surgidos del contexto, pero la pluralidad de modelos de método que iba apareciendo en el ámbito del diseño auguró que no había soluciones simples para los entornos cada vez más complejos y que se debió revisar y replantear el papel que desempeñaban los métodos en el diseño y los enfoques que los sustentaban.

Esta hipótesis que defiende Rodríguez, sobre la utilidad que ofrecerían los métodos en diseño para manejar la creciente y confusa información devenida del contexto, queda probada por la misma estructura de los modelos que florecieron en los años 60 y 70 del siglo anterior; así el trazado proyectual iniciaba con la especificación del problema, fase adoptada de los esquemas de investigación tradicional y de las ciencias naturales, situación normal en un entorno en el que el diseño estaba marcado por un afán de racionalidad y objetividad que se identificaba en los modelos de este tipo de ciencias. A modo de ejemplo, Rodríguez (1990) menciona las distintas conferencias que abordaron el tema del método:

de esta manera se fue produciendo una gran inquietud por los métodos en diseño y diversas opiniones se fueron sumando hasta desembocar en 1962 en una conferencia sobre métodos de diseño en Londres. La preocupación en esta conferencia fue el diseño "sistemático" con un énfasis especial en las técnicas. En la década del sesenta se realizaron más conferencias sobre el tema. Destacaron la de 1965 en Birmingham (Inglaterra) titulada "el método de Diseño", donde se realizó un claro intento por buscar puntos comunes entre el método científico y el diseño; sin embargo, al final de la conferencia no se obtuvo un claro consenso al respecto. (p. 32) 
Evidenciado el afán racionalista en el diseño, la importación de procedimientos de las ciencias naturales solo se preocupó por la incorporación de estrategias para especificar algunas etapas del proceso y el diseño olvidó —o no supo interrelacionar- los enunciados de las teorías de las que devenían tales estrategias; lo que deja a la actividad proyectual con vacíos de tipo teórico e incluso lógico, los mismos que han exigido la anexión posterior de tácticas y técnicas de diversas áreas.

En este sentido los cambios en la estructuración de los modelos de método atendían, por lo regular, a verificar si se procedía en relación a la noción de "caja negra" o de "caja transparente"; conceptos muy aprehendidos en las reflexiones sobre los métodos, que en general se pueden describir por la posibilidad de ofrecer garantías sobre el resultado y la condición de demostrar la razón que asiste al diseñador para haber logrado el resultado específico y no otro. Este tipo de caracterizaciones de "caja negra" y "caja transparente", más que modelos, son formas en las que el diseñador actuaba respecto a la organización de las tareas que implicaba el acto de diseñar; por supuesto, la más confiable es el proceder mediante "caja transparente" que está mediada por diversas acciones que implican entre otras cosas la previsión y la consciencia de la temporalidad que caracterizan al diseño. Rodríguez (1990) describe sus características:

1. Objetivos, variables y criterios de evaluación son claramente fijados de antemano.

2. El análisis del problema debe ser completado antes de iniciar la búsqueda de soluciones.

3. La evaluación es fundamentalmente verbal y lógica (en lugar de experimental).

4. Las estrategias se establecen de antemano.

5. Por lo general las estrategias son lineales e incluyen ciclos de retroalimentación. (p. 33) 
Ahora bien, estas características están relacionadas por ambas categorías que describen el proceso de diseño: la previsión y la consciencia de la temporalidad, que enlazan los criterios fijados de antemano como son el análisis y las estrategias previas; en otras palabras, el modo de trabajar de la "caja transparente" contiene la idea de un proceso que posee la capacidad de demostración pretendida por ese afán de racionalidad que caracterizó al diseño en el período de tiempo citado.

La previsión y la temporalidad del diseño se mencionan con mayor énfasis en la obra de Martí Font (1999):

els artefactes, abans de la seva producción que els incorpora al món real, en contrast amb la natura però derivats d'aquesta, tenen una realitat prèvia imaginada en la ment d'algú. Són el resultat dún procés de previsió. No podrien existir sense aquest procés [...]. El disseny és una activitat que se desenvolupa en el temp i en conseqüència ha de ser contemplat també des del put de vista del seu procés. (p. 77) ${ }^{1}$

Ambas consideraciones de Martí Font manifiestan de plano que el diseño es una actividad atravesada por el tiempo y, en consecuencia, con la implicación de la previsión; esto significa que deben estar presentes en la mente del diseñador y se convierten en características que los métodos en diseño deben poseer para aclarar la estructura del proceso proyectual. Sin embargo, los modelos de método utilizados en el diseño, si bien asumen la previsión y la temporalidad como características del proceso, parecen preocuparse por identificar la aparente regularidad que emerge de las acciones que se siguen para la obtención de un artefacto y por establecer con claridad la cercanía entre la idea inicial y el resultado pretendido.

\footnotetext{
1 "Los artefactos, antes de su producción que los incorpora al mundo real, en contraste con la naturaleza pero derivados de ella, tienen una realidad previa imaginada en la mente de alguien. Son el resultado de un proceso de previsión. No podrían existir sin este proceso [...]. El diseño es una actividad que se desarrolla en el tiempo y en consecuencia ha de ser contemplado también desde el punto de vista de su proceso". Traducción libre por parte del autor.
} 
De ahí que, a pesar de que se han elaborado innumerables modelos de método desde los años 60 y 70 del siglo pasado hasta ahora, es necesario advertir que la revisión de estos no se cubre en el presente escrito; no obstante, sí se puede concluir con ello que se siguen persiguiendo las consideraciones que en su momento les dieron origen: los modelos de método de donde provengan tienen claro que sus tareas son la reducción de la complejidad del proceso, revisión de la lógica interna del acto de diseño y la comprensión de la estructura del proceso proyectual.

Con estas ideas en mente se quiere acercar el presente estudio al contexto inmediato, el cual posee variadas ideas sobre lo que significa el término diseño; aunque para acercarnos a alguna de ellas, y revisar en parte su vinculación con el contexto en historia del diseño en América Latina y el Caribe, Silvia Fernández y Gui Bonsiepe (2008) recopilan una serie de ensayos críticos en los que se hace mención sobre la disciplina del diseño en Colombia:

el diseño nació como una apuesta por modernizar el sector empresarial, por contribuir a introducir las ideas modernas en el pensamiento y la cultura presentes en el aparato productivo colombiano, o bien como una apuesta por introducir la cultura del proyecto en la cultura colombiana. (p. 90)

En esta concepción del diseño se pueden apreciar ideas que no es conveniente dejar de mencionar: primero el diseño se presenta como un proceso que permite modernizar —es decir que está atado a la idea de modernidad, a las ideas modernas, al pensamiento y a la cultura productiva que se deriva de la Modernidad-, pero tal vez no se pensó mucho en la importación de los modelos de los que venía acompañado —es decir que se quiso implementar una idea de modernidad en un contexto que, como bien se expone en algunos apartes del texto citado, estaba aún muy enlazado a mecanismos y procesos característicos del feudalismo-. 
Por su parte la inserción en el contexto inmediato también desempeña un papel de propagador de la cultura del diseño en la sociedad colombiana, lo que implica que después los diseñadores se convirtieran en quienes dictan los modos en los que actúa el diseño en la sociedad; aunque no desde el análisis de las necesidades contextuales de la misma sino como un intento de imitar otros entornos en los que el diseño se asentó hace mucho más tiempo y tuvo una evolución un poco más estable.

Ese afán de modernizar el sector productivo e introducir la cultura del diseño al contexto deja en claro que todo lo demás se importó, tanto las teorías del diseño como los métodos en diseño; de ahí que este trabajo empieza por delinear un primer acercamiento a un procedimiento que responda de alguna manera a esas exigencias que el mismo contexto manifiesta.

\section{Primeros acercamientos para la propuesta DVUC-001}

La complejidad que encierra la proposición de un modelo de método para el diseño solo se hace evidente en la medida en que se advierte la relación - muchas veces oculta o ignorada- entre el método, la teoría y el acto de diseño. Esta relación implica necesariamente que se trate de esquematizar lo más representativo del proceso de diseño, entendido como paso del tiempo; es decir como el paso de un punto $A$ a un punto $B$, movimiento que deviene en una transformación. 


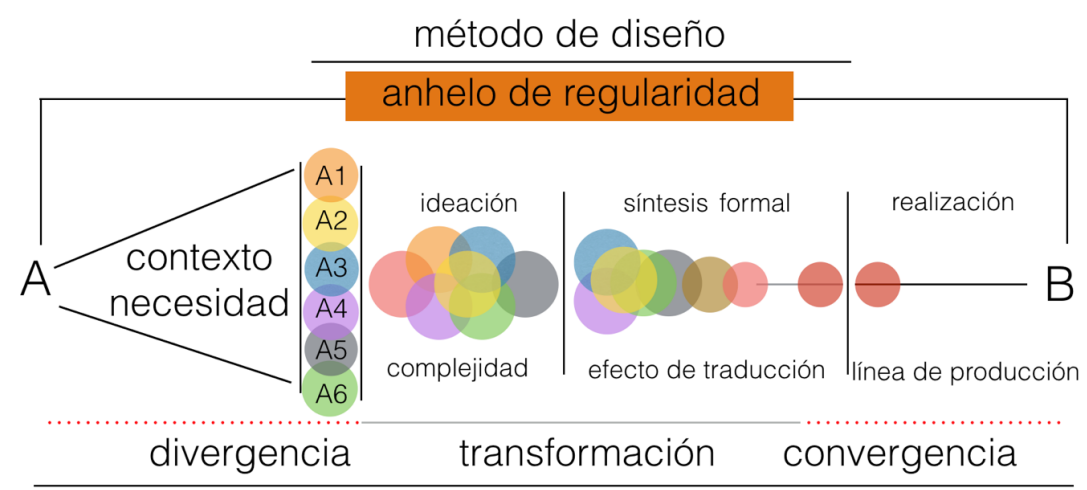

REDUCCIÓN DE LA COMPLEJIDAD - REVISAR LA LÓGICA INTERNA DE LA SECUENCIA DEL ACTO DE DISEÑO -

COMPRENDER LA ESTRUCTURA DEL PROCESO PROYECTUAL.

teoría de diseño

Figura 1. Relación entre la estructura del proceso de diseño, el método en diseño y la teoría del diseño. Fuente: elaboración propia por parte del autor.

La figura 1 intenta mostrar algunas de esas constantes en el proceso general del diseño para evidenciar esa relación entre la teoría y el método en diseño, pero para dar paso a la explicación de esta figura es necesario hacer referencia a la idea de regularidad que asiste a un proceso como el del diseño; pues existe una característica que marca la reflexión sobre los métodos en diseño y es que la eficiencia de los modelos metodológicos en diseño se basa en la consideración de que en el proceso proyectual hay una estructura común, hay elementos constantes que permiten abordar los diversos problemas, haciendo abstracción de sus particularidades y poniendo en acto la acción de diseñar. Estas reflexiones dejan en claro que existe cierta confianza al momento de abordar un nuevo problema de diseño, es decir, que se comporte de manera parecida a los ya resueltos y que de ahí el método sea eficiente en su resolución. Sin embargo, nada puede asegurar que los problemas actúen de 
maneras similares, esto es: "este hecho de la regularidad existe porque hay una expectativa de regularidad. Después de todo, el término de irregularidad tiene sentido sólo si disponemos de una regla" (Feyerabend, 1975, p. 106).

Volviendo a la figura 1 se observan un punto $A$ y un punto B, los cuales representan el inicio de un proceso y el logro después de cumplir con el mismo; estos puntos están unidos por ese anhelo de regularidad que los métodos entrañan. El contexto y la necesidad, ambos términos recurrentes en los estudios de diseño, se colocan después del punto de inicio dado que de ellos dependen una serie de posibilidades de partida y son representados con la denominación A1, A2 ..., A6; estos acontecimientos emergen del contexto o del estudio de la necesidad particular, etapa generalizada de los procesos metodológicos en diseño.

De acuerdo con la linealidad del proceso en diseño se pasa a las fases de ideación, síntesis formal y realización, las cuales poseen ciertas caracterizaciones que se han establecido como "lugares comunes" en la valoración de la dificultad de trabajar en el diseño; es decir la ideación es considerada como una de las etapas con alto grado de complejidad; la síntesis formal se caracteriza por ser una traducción formal de los requerimientos más sobresalientes de la necesidad o problema entretanto la realización, si bien puede presentar sus propias dificultades, se relaciona con una línea de producción específica de cada una de las áreas del diseño ya sea industrial o gráfico. Estas caracterizaciones sobre las tres etapas del proceso de diseño se representan con círculos de colores que van simplificándose hasta quedar uno solo en la etapa de realización, el cual está unido al punto B como manera de significar que se ha obtenido el resultado.

Existe otra manera para referirse a las etapas del proceso de diseño que obedecen a los enfoques que se hacen al proceder en la acción de diseño, 
la divergencia a la que se enfrenta el diseñador al momento de valorar la necesidad y la información devenida del contexto; la transformación de esa información que sucede en su mente y que converge en un resultado en el que, al parecer, toda la complejidad se ha simplificado.

Finalmente, en la figura 1 se enuncian los objetivos pretendidos por el método en diseño, reducir la complejidad y comprender la estructura del proceso y revisar la lógica interna de la secuencia del acto de diseño, los cuales en algunas ocasiones han sido tareas que se establecen para la teoría del diseño puesto que al lograr estos objetivos se están comprendiendo las problemáticas con las que se enfrenta el diseño.

En otras palabras, los aspectos que definen un método - por lo regular- se fundamentan en una teoría que permita su articulación; es decir que el método responde a una teoría que involucra y justifica técnicas y que establece sus posibilidades o limitaciones respecto al trabajo sobre su objeto, el mismo que se encuentra delimitado por la teoría a la que refiere. En el caso del diseño los métodos con los que ha trabajado presentan diversas vinculaciones con teorías ubicadas entre las ciencias naturales, las filosóficas, hasta los enfoques de tipo social. Bürdeck (2002) lo expresa de esta manera:

si inicialmente, el paso del paradigma de las ciencias naturales al de las ciencias filosóficas ha sido incorporado al diseño como hipótesis, desde hace unos años han aparecido nuevas orientaciones. Por un lado se encuentra la tradición del diseño conceptual de los años sesenta en Italia, con la que conectan diversos grupos de diseñadores como Kunstflug o GINBANDE. Desde un punto de vista metodológico, pasan ahora a un primer plano las interconexiones, las referencias cruzadas y las asociaciones sobre cuyas bases se desarrollan soluciones de diseño nada convencionales. La creciente complejidad de los encargos profesionales (p.e., la comunicación en la oficina, la electrónica en la vida diaria, los problemas de transporte de masas o las consecuencias ecológicas de un proyecto), remite entre otras cosas a un nuevo significado de la metodología. Tras el "Design aus dem Bauch" (Diseño visceral) se trabaja ahora de nuevo en temas de orientación social. (p. 166) 
En este panorama se hace complejo establecer una relación clara entre los métodos de diseño y las teorías de diseño dado que en muchas ocasiones el cuerpo teórico del diseño se ha confundido con interconexiones entre saberes y otras veces con referencias a otros campos que no permiten una revisión sobre el tipo de objeto que la teoría del diseño debería describir; no obstante, esto no será tema del presente escrito. En lo referente a la esquematización realizada se resalta la necesidad de hacer posible el reconocimiento de los aspectos que son regulares en la obtención de una pieza de diseño, ya que en la actividad proyectual coinciden una serie de procedimientos que son frecuentes y que están presentes en las maneras de pensar y hacer de los diseñadores; sin embargo esta regularidad, si bien se advierte en la superficie del proceso, parece que no hay una manera de asegurar un correcto orden a los criterios que se desprenden del análisis de las problemáticas que dan origen a un proyecto para su paso a la síntesis formal que se requiere.

Estas inquietudes han estado presentes a lo largo de los estudios de la actividad proyectual, lo que ha llevado a la proliferación de diversos modelos de método en diseño y la imposibilidad de trazar un modelo unificado que permita la articulación de los posibles principios inscritos en su teoría a los diversos problemas que atañen a la disciplina del diseño; la búsqueda de tales principios ha estado mediada por interconexiones y referencias a otras áreas del conocimiento que permiten establecer en algún punto claridad sobre el proceso de diseño. Por otro lado, esta ausencia de unificación, más que un obstáculo en la realización de proyectos de diseño, se ha convertido en la oportunidad de expresar lo contextual como la diferenciación que hace parte de los objetos de diseño en sus diversas ramas.

El método en diseño, al entenderse como un "esquema racional trazado por las líneas de acción que deben seguirse para la construcción de las ideas" (González Ruiz, 1994, p. 60), implica una caracterización sobre la producción 
de conocimiento; en otras palabras, al ser racional, se espera que al utilizar el método en diseño se produzca un conocimiento respecto del proceso de diseño o del artefacto obtenido o de los principios de alguna de las teorías que intentan delinearlo; así, se hace evidente una ideología que subyace a los modelos y a la manera en la que se concibe el diseño.

\section{Algunos aportes disciplinares al modelo DVUC-001}

Inicialmente se ha tomado a la imagen en su relación con el diseño, es decir en el momento inicial de la investigación se consideró que la imagen es un instrumento de comunicación que tiene una estrecha relación con el diseño de comunicación visual dado que las piezas que se obtienen en este tipo de diseño tienen por finalidad comunicar, establecer relación con su usuario, transmitir informaciones e incidir en sus decisiones. La imagen ha sido objeto de diversas reflexiones en el ámbito de la comunicación, pero en el diseño aparentemente ha quedado inscrita dentro de la funcionalidad estética o lo que es lo mismo la imagen se ve como una parte resultante de la planificación formal.

Desde su propia teoría, la imagen posee tantas o más dificultades en su formalización que el diseño; aún no alcanza los estatus que se exigen desde las corrientes epistemológicas más tradicionales para su configuración como una teoría autónoma. Sin embargo, esfuerzos como los de Villafañe y Mínguez (1996) y los de Sanz (1996) han permitido que la imagen posea planteamientos que permitan explicar algunas de las manifestaciones de la comunicación visual. Ambos trabajos hacen parte del primer acercamiento al modelo DVUC001, al considerar que la imagen en su relación con el diseño de comunicación visual adquiere una manifestación de lenguaje y actúa como

un sistema específico de formas cromáticas - autoluminosas o dependientes-, producidas por el artífice a través de los procedimientos, materiales y utensilios propios de cierto medio, las cuales percibe el espectador a través de su aparato visual —psicofisiológico-, siendo la transmisión de 
un mensaje, la construcción de imágenes significativas en el espectador, su principal finalidad y, de modo secundario, la exteriorización del propio pensamiento visual o de los propios sentimientos de artífice. (Sanz, 1996, p. 45)

Esta consideración sobre el lenguaje de la imagen lo equipara al diseño respecto a su principal finalidad, trasmitir un mensaje y construir imágenes significativas en la mente del espectador; por ello la dinámica implícita de su sistema se retoma para articularlo en las primeras fases del modelo propuesto, mientras que lo proyectual se manifiesta como el proceso con el cual se pretende la comunicación; con esta premisa clara, los principios o nociones que han servido de base para la formulación del modelo se recapitulan de las teorías más representativas de la imagen, la comunicación y el diseño.

Desde las teorías de la imagen sobresalen nociones como selección de la realidad, repertorio de elementos de representación, sintaxis y funciones de realidad de la imagen, las cuales son retomadas de Villafañe y Mínguez (1996). La noción de función de realidad de la imagen se retoma de autores como Arnheim (1978), Aumont (1992) y Villafañe y Mínguez (1996). En este mismo espectro se consideran conceptos como el lenguaje de la imagen, elementos del lenguaje de la imagen, mensaje visual, código icónico, los cuales se recopilan de los estudios de la imagen que hace Sanz (1996).

A partir del área de la comunicación y la subárea de la comunicación visual, las nociones que se utilizaron quedaron en mayor parte subsumidas en el proceso mismo del diseño de la comunicación visual que adquiere las características del proceso comunicativo específico; por ello es posible mencionar dos: la noción de retórica de la imagen (Barthes, 2009) donde se establecen los aspectos de los tres modos en los que se presentan los mensajes visuales, mensaje icónico no codificado, mensaje icónico codificado y mensaje lingüístico. La comunicación visual y las figuras retóricas se recogen desde Guillermo González Ruiz (1994) al interior de los estudios del diseño. 
De los estudios de diseño se usaron diversas nociones que, desde varios autores, permiten revisar al diseño como proceso tanto de formalización de piezas como de comunicación; además de algunas concepciones que brindan piso a la noción de diseño con fines comunicativos. Una de las nociones más interesantes y que permite la ubicación de la actividad proyectual en la comunicación es la de diseño como acto relativo a un contexto; la noción que deviene desde Enrique Dussel (1984), enmarca al diseño como una actividad humana al interior de una serie de determinantes que condicionan su desarrollo; de ahí que tomar al diseño como contextual permite su ubicación en un entorno, el cual marca a la actividad proyectual según las condicionantes que existen en él.

Por su parte el diseño como proceso racional y sistemático (Archer, 1968) permite la correspondencia entre el diseño y el proceso de comunicación de manera más naturalizada, además de aportar cierta facilidad para la estructuración del modelo.

El diseño, en la concepción de Jorge Frascara (2006), es un proceso de planificación para conseguir un propósito; esto permite asegurar su diferenciación con otros procesos de tipo creativo, por lo que se remarca la intencionalidad que lo caracteriza y de paso hace evidente la vinculación con la previsión de la que se habló en líneas anteriores. Otro de los puntos interesantes que se extraen de las concepciones de Frascara (2006) es la de situación comunicacional, "el objetivo del diseñador de comunicación visual es, entonces, el diseño de situaciones comunicacionales" (p. 31), concepto que establece claramente la finalidad del ejercicio del diseño: la interacción entre el mensaje y el usuario.

En cuanto al diseño como proceso limitativo se puede afirmar que no todo proceso creativo es diseño, así para González Ruiz (1994) es fundamental 
establecer estas diferenciaciones entre los procesos de tipo creativo debido a que en el imaginario el diseño tiene que ver con una libertad creativa; de ahí que se deba establecer cierto tipo de control que va desde la ideación hasta la realización, lo que implica la previsión que deriva de la temporalidad; sin embargo muchos de los postulados sobre la creatividad y el diseño hoy dan por sentado que la libertad creativa es el núcleo central del proceso de diseño y hacen una reivindicación de los estados intuitivos para el logro de resultados, pero esta crítica no se hará en los límites de este escrito solo basta decir que el modelo propuesto implica la necesidad de control antes que la vuelta a la "caja negra". Por otro lado, también se revisaron dos nociones sobre la teoría del color, particularmente el enfoque de Moles y Janiszewski (1992), las cuales quedan incluidas en el aspecto cromatológico derivado de la noción del lenguaje de la imagen.

Ahora bien, la configuración de un modelo intenta representar un sistema real que en este caso sería la acción de diseñar; es decir aquello que debe guiar el ejercicio de efectuación en la actividad proyectual en la realidad, el cual expresa la interacción de las partes que se representan en el modelo; de ahí que sea necesario pasar a determinar la manera en la que la situación comunicativa se inscribe en el procedimiento propuesto; de esta forma el modelo DVUC001 sintetizó una serie de posiciones teóricas alrededor de la imagen como instrumento comunicativo, lo que permitió la vinculación de algunos de los elementos del lenguaje de la imagen para facilitar la incorporación de sus funciones en la construcción de los primeros bocetos en el trazado proyectual derivado de su aplicación.

Si bien es claro que el desarrollo de una pieza de diseño de comunicación visual tiene como fin último el establecimiento de significaciones en la mente de los usuarios, esta situación hace parte de un conjunto mucho más amplio de relaciones en el escenario de la realización de diseño. Por ello, 
y en correspondencia con la noción de contexto, se denominaron las otras situaciones por las que atraviesa el proceso de diseño como: situaciones de partida; situación proyectual; situación comunicativa y situación gráfica; esta última haciendo alusión al soporte físico en el que una interacción comunicativa toma cuerpo. Estas situaciones se entienden como contextos en los que el diseñador se relaciona de diversa manera con la problemática que intenta solucionar.

Estas situaciones se equiparán a los momentos mejor entendidos que componen el proceso de diseño, a saber: la situación de partida se correlaciona con la fase de conceptualización-análisis; la situación comunicativa se relaciona con la fase de conceptualización-síntesis; la situación proyectual tiene relación con la fase de formalización y la situación gráfica obedece a las características de la fase de realización en el diseño. Desde allí, al proponer esta correlación entre situaciones y fases del proceso de diseño, se evidencia que el modelo DVUC-001 debe estar compuesto por estrategias que obedezcan y sigan la lógica de esa relación. En ese mismo orden de ideas, se ubicaron 11 momentos: encargo del proyecto; recolección de información; primera visualización; segunda visualización; evaluación estructural; primera jerarquización; tercera visualización; evaluación cliente; segunda jerarquización; cuarta visualización; producción. Cada uno de estos momentos tiene una guía para ser aplicado y se describe claramente la acción que debe seguir el diseñador.

Se puede apreciar en la figura 2 la esquematización lineal del modelo donde las once estrategias están listadas en la segunda columna de la izquierda, enmarcadas por las situaciones procedimentales en la primera columna de la izquierda y por los momentos del proceso de diseño; los cuales están descritos en la última columna a la derecha con la intención de que funcionen como una ubicación para su fácil aplicación, es decir que las situaciones en las que se encuentra el diseñador durante la aplicación del método se corresponden 
con estadios más reconocidos del proceso de diseño; de tal manera que la ubicación en las situaciones ayude a identificar las transformaciones del problema a resolver.

\begin{tabular}{|c|c|c|c|c|c|c|}
\hline \multicolumn{7}{|c|}{ DVUC-001 } \\
\hline $\begin{array}{l}\text { SITUACIÓN } \\
\text { PROCEDIMENTAL }\end{array}$ & & & & & & PROCESO DE DISEÑO \\
\hline $\begin{array}{l}\text { Relación dialógica } \\
\text { con el cliente }\end{array}$ & $\begin{array}{l}\text { ENCARGO DEL } \\
\text { PROYECTO }\end{array}$ & & & & & \\
\hline \multirow{3}{*}{$\begin{array}{l}\text { SITUACIÓN DE } \\
\text { PARTIDA }\end{array}$} & $\begin{array}{l}\text { RECOLECCION DE } \\
\text { INFORMACIÓN }\end{array}$ & CROMATOLÓGICO & CONFIGURATIVO & ICONOGRÁFICO & TIPOGRÁFICO & \multirow[b]{2}{*}{$\begin{array}{l}\text { CONCEPTUALIZACIÓN - } \\
\text { ANÁLISIS }\end{array}$} \\
\hline & $\begin{array}{l}\text { DEFINICIÓN DE } \\
\text { OBJETIVOS } \\
\text { GENERAL (Encargo) } \\
\text { ESPECífICOS - } \\
\text { (Cromatológico, } \\
\text { configurativo, } \\
\text { iconográfico, } \\
\text { tipográfico) }\end{array}$ & $\begin{array}{l}\text { USOS } \\
\text { CROMÁTICOS } \\
\text { CONVENCIONAL } \\
\text { CULTURAL }\end{array}$ & $\begin{array}{l}\text { NATURAL } \\
\text { ESQUEMA } \\
\text { ABSTRACTO }\end{array}$ & $\begin{array}{l}\text { SIGNO } \\
\text { REPRESENTACIÓN } \\
\text { SIMMBOLO }\end{array}$ & $\begin{array}{l}\text { CAPACIDAD } \\
\text { EXPRESIVA } \\
\text { AUMENTADA } \\
\text { LEGIBILIDAD } \\
\text { REDUCIDA } \\
\text { CAPACIDAD } \\
\text { EXPRESIVA } \\
\text { REDUCIDA } \\
\text { LEGIBILIDAD } \\
\text { AUMENTADA } \\
\end{array}$ & \\
\hline & $\begin{array}{c}\text { PRIMERA } \\
\text { VISUALIZACIÓN }\end{array}$ & \multicolumn{4}{|c|}{$\begin{array}{l}\text { ELECCIÓN CONTEXTUAL DE COMPONENTES } \\
\text { ESTRUCTURA BÁSICA (SUBJETIVO) }\end{array}$} & \multirow{3}{*}{$\begin{array}{l}\text { CONCEPTUALIZACIÓN - } \\
\text { SINTESIS }\end{array}$} \\
\hline \multirow{5}{*}{$\begin{array}{l}\text { SITUACIÓN } \\
\text { COMUNICATIVA } \\
\text { PROYECTUAL }\end{array}$} & $\begin{array}{l}\text { SEGUNDA } \\
\text { VISUALIZACIÓN }\end{array}$ & \multicolumn{4}{|c|}{ ESTRUCTURA BÁSICA FORMAL (DECISIÓN) } & \\
\hline & $\begin{array}{l}\text { EVALUACIÓN } \\
\text { ESTRUCTURAL }\end{array}$ & \multicolumn{4}{|c|}{$\begin{array}{l}\text { VALORACIÓN DE CÓDIGOS / CROMÁTICOS - ICÓNICOS - LINGÜISTICOS } \\
\text { CATEGORIAS GENERALES DE COMUNICACIÓN - DESEMPENOO DEL PROYECTO }\end{array}$} & \\
\hline & $\begin{array}{l}\text { PRIMERA } \\
\text { JERARQUIZACIÓN }\end{array}$ & COLOR & TECNICAS DE & EPRESENTACIÓN & FUENTES & \multirow{4}{*}{ FORMALIZACIÓN } \\
\hline & $\begin{array}{l}\text { TERCERA } \\
\text { VISUALIZACIÓN }\end{array}$ & \multicolumn{4}{|c|}{$\begin{array}{l}\text { ESTRUCTURA COMUNICATIVA / INTENCIÓN COMUNICATIVA / MENSAJE ICÓNICO } \\
\text { VERBAL }\end{array}$} & \\
\hline & $\begin{array}{l}\text { EVALUACIÓN } \\
\text { CLIENTE }\end{array}$ & & CORRESPOND & NCIA OBJETIVOS & & \\
\hline \multirow{3}{*}{$\begin{array}{l}\text { SITUACIÓN } \\
\text { GRÁFICA }\end{array}$} & $\begin{array}{l}\text { SEGUNDA } \\
\text { JERARQUIZACIÓN }\end{array}$ & \multicolumn{4}{|c|}{ CUATRO PROPUESTAS SEGÚN COMPONENTES } & \\
\hline & $\begin{array}{l}\text { CUARTA } \\
\text { VISUALIZACIÓN }\end{array}$ & \multicolumn{4}{|c|}{ DOCUMENTOS - PLANOS- DEFINITIVOS } & \multirow{2}{*}{ REALIZACIÓN } \\
\hline & PRODUCCIÓN & \multicolumn{4}{|c|}{ ARTES FINALES/SUPERVISIÓN } & \\
\hline
\end{tabular}

Figura 2. Esquematización lineal del modelo DVUC-001. Fuente: elaboración propia por parte del autor. 
En las columnas del centro se aprecian los aspectos derivados de los enfoques teóricos más relevantes. Luego de entender que el encargo del proyecto primera fase- es una relación dialógica con el cliente, la segunda fase es la recolección de información compuesta de la definición de objetivos general y específicos; estos objetivos se relacionan con los elementos del lenguaje de la imagen tales como lo cromatológico, configurativo e iconográfico, y la desagregación de estos elementos - es decir que se presentan las caracterizaciones de color, los principios constituyentes de las figuras y las categorías icónicas dentro de un aspecto visual determinado-. Por su parte el componente tipográfico, si bien no pertenece al lenguaje de la imagen, se incluye en esta fase para que su desarrollo sea equivalente y simultáneo con el componente icónico y cromático dentro del cual se presentan las maneras en las que actúa según su capacidad expresiva y su nivel de legibilidad.

La tercera fase del modelo, la primera visualización, retoma la idea de la contextualización en la medida en que propone la elección de compontes adscritos a las categorías descritas en la primera fase desde el contexto en el que se identifica el problema. Esta fase tiene un componente altamente subjetivo.

La cuarta fase, la segunda visualización, se enfoca en la obtención de una idea general visualizada con la idea de tomar una decisión sobre la interrelación de los componentes icónico, cromático y tipográfico. En esta fase prima una consideración de conjunto de la pieza.

En la quinta fase, evaluación estructural, se revisa la actuación de los componentes respecto de los códigos icónicos cromáticos y lingüísticos en relación con la estructura comunicativa. En este punto se revisa el desempeño del proyecto según los objetivos trazados y su cumplimiento como pieza de comunicación visual. 
Sexta fase, primera jerarquización. Al observar el comportamiento de la pieza de comunicación —en relación a los objetivos - se pasa a considerar la participación y protagonismo que el color, las técnicas de representación icónica y las fuentes tipográficas tienen en la pieza para transmitir efectivamente el mensaje.

Séptima fase, tercera visualización. Si se hace necesario se reordenan los componentes icónico, cromático y tipográfico con miras a ajustar la estructura comunicativa, su intencionalidad y la coherencia entre el mensaje icónico y las expresiones verbales que lo acompañan.

Octava fase, evaluación cliente. En esta fase se revisan los objetivos planteados y la relación con las diversas propuestas que se han elaborado en las etapas de visualización anteriores, de ella se desprende una segunda jerarquización: la novena fase; que permite la elaboración de propuestas acordes con los resultados de la evaluación del cliente y el énfasis en cada componente, icónico, cromático, tipográfico o la estructura equipotencial de todos ellos.

Décima fase, cuarta visualización. Esta es la propuesta definitiva en la que se desarrollan los documentos y planos definitivos para la producción.

Undécima fase, producción. Enviar artes finales y proceso de supervisión de la producción.

Cabe resaltar que en el modelo propuesto se tuvo en cuenta la reiteración, la cual correlaciona las fases de evaluación estructural con la fase de recolección de información; la evaluación del cliente con la evaluación estructural y finalmente la segunda jerarquización con la fase de tercera visualización. Estos ciclos de reiteración buscan la depuración del trabajo y el control a partir de los objetivos trazados. 
Al ser un modelo enfocado en el área del diseño gráfico o de comunicación visual, la visualización cobra gran importancia; es decir que el diseñador debe estar en continua producción visual, corroborando la traducción que implica la formalización y posteriormente el refinamiento de las propuestas; mientras que la jerarquización hace alusión a la estructuración de la pieza mediante la valoración de los componentes principales, color, imagen y texto para de esa manera considerar la implicación de la comunicación en el proceso.

\section{Metodología}

El estudio inició con una caracterización cercana a lo correlacional, ya que determina la relación entre variables. La situación comunicativa, como elemento constitutivo del modelo, se relaciona con la depuración en las realizaciones de los diseñadores del área gráfica por la aplicación del modelo DVUC-001; de igual manera esta caracterización de la investigación implicó una prueba piloto, la cual buscaba información sobre la estructura del modelo propuesto después de su aplicación para obtener información sobre la comprensión de las variables que influyen en el diseño de una pieza gráfica mediante la utilización del modelo.

Se establecieron tres momentos de observación: un primer cuestionario, no estructurado y de preguntas abiertas, antes de iniciar el trabajo con el modelo desarrollado; un segundo momento que es la inspección en el instante de aplicación del modelo a los estudiantes y un tercer momento que es un cuestionario, de iguales características que el primero, realizado después de la aplicación del método propuesto.

Estas observaciones dan porcentajes sobre lo que interesa en la construcción del modelo; es decir las ventajas y desventajas en su utilización, la pertinencia de un modelo metodológico en diseño y los aspectos que debería ofrecer un 
método en diseño para ser utilizado por los estudiantes. Estos tres elementos sintetizan los objetivos buscados en el estudio, después de presentar el modelo DVUC-001. Respecto a la muestra esta fue seleccionada no probabilística intencional, por considerar la disponibilidad de estudiantes para efectos de la aplicación del modelo.

\section{Resultados}

El procesamiento de los datos intentó organizar las categorías comúnmente usadas en los modelos de métodos en diseño y describir la relación establecida por los estudiantes con el modelo desarrollado. Así pues, al recibir las respuestas a los cuestionarios, se buscó la regularidad de los factores que inciden en las ventajas y desventajas en la utilización de los modelos de método en general y en el modelo desarrollado en particular. Los porcentajes establecidos solo pretenden ordenar los términos usados por los estudiantes en relación a la facilidad de aplicación del modelo. Los participantes tuvieron la posibilidad de agruparse o de trabajar individualmente, lo que permitió comparar el logro de las tareas y la facilidad que ofrece el modelo propuesto.

Sobre las ventajas en la utilización de métodos en diseño, los participantes señalaron trece aspectos entre los cuales destacan: la ventaja que tiene el método como una guía racional con un 23,26 \%, es la categoría que más acuerdo obtuvo en los estudiantes. La reiteración, entendida como la posibilidad de retroceder en la secuencia para corregir errores, obtuvo un porcentaje del 13,96 \%. La recolección de información y la posibilidad de usar el método en diversos formatos visuales, cada una arrojó un 11,62 \%. La flexibilidad que caracterice la estructura del método con un 9,30 \%, completa los valores más altos y con mayor acuerdo.

En relación a las desventajas se enumeraron siete en total, de las cuales emergen cinco con mayor porcentaje: la primera caracteriza al tiempo requerido en la 
utilización y aplicación de métodos en los períodos de clase, es decir que el tiempo de clase no es suficiente para la aplicación correcta de este tipo de modelos, alcanzando un $25 \%$. Otro factor negativo es que al ser racional los modelos reducen la creatividad, entendida como la libertad formal para solucionar problemas de diseño con un 20,80 \%. La poca flexibilidad en las estructuras de los métodos es otra de las desventajas que se desprende del cuestionamiento con un $16,70 \%$, alcanzado un alto porcentaje en las respuestas. Dos aspectos que se identificaron como desventaja, ambos con $12,50 \%$, son la redundancia en las fases de los métodos y la discrepancia entre la academia y el medio laboral; sobre la primera se puede enunciar que los estudiantes identifican que, en la aplicación de los métodos, existe cierta duplicación de fases o que algunas de ellas sobran; mientras que sobre la discrepancia entre la academia y el medio laboral, los estudiantes reconocen que la aplicación de métodos no siempre se puede dar en el medio laboral, dejando a los métodos solo como exigencia de la academia.

Respecto al cuestionamiento sobre los aspectos que debe tener el modelo para ser utilizado por los estudiantes, los participantes expresaron en un mayor porcentaje que debe tener una estructura de corte investigativo con un 45,40 $\%$; mientras que la estructura comunicativa que debe estar presente en el modelo, obtuvo un $27,30 \%$. Luego aparecieron otros aspectos generales que se concentran en tres elementos: una regularidad que funde el trabajo intuitivo, la pertinencia y la practicidad del modelo y la posibilidad de estructurar el pensamiento de diseño, alcanzando cada una un 9,10\%.

Ya en el proceso de aplicación del modelo se recurrió a la observación, que aporta aspectos de tipo descriptivo guiados por algunas categorías que intentan verificar la manera en la que el método permite cierta interacción con las tareas asignadas. Es necesario precisar que, para este momento, se elaboró un enunciado que presentaba un problema de comunicación visual que se 
debía resolver mediante la aplicación del modelo DVUC-001 y entregar piezas gráficas. Entre las categorías de observación se encontraban:

1. Gestión del tiempo: esta categoría buscaba la organización por parte de los estudiantes de las etapas básicas que permiten diferenciar entre los momentos para la obtención del logro: inicio, desarrollo y cierre; de esta categoría se aprecia que los estudiantes — si bien iniciaron con la tarea en el laboratorio- la mayor parte de ellos alcanzó a cumplir la primera etapa, inicio, especialmente en la recolección de información y en la caracterización del grupo objetivo. Las otras etapas, desarrollo y cierre, se surtieron por fuera del laboratorio pero bajo la supervisión del equipo de investigación.

2. Implementación: en la realización del ejercicio se pudo apreciar que el modelo DVUC-001 permite la participación activa de los estudiantes al estar agrupados; asimismo, una interdependencia positiva y la delegación de responsabilidades concretas a cada uno de los integrantes. En contraste, al trabajar de manera individual, el modelo ofrece un seguimiento consecutivo que resulta similar al que otros modelos permiten.

3. Calidaddelasinstrucciones:el modeloDVUC-001 ofreceuna instruccionalidad clara y precisa, lo que se refleja en las once estrategias que lo componen y que los estudiantes siguieron de manera lógica y secuencial para obtener los logros que requiere la tarea. Se manifestaron algunos inconvenientes con la utilización de términos, como jerarquización y visualización, que requirieron explicación adicional por parte de un docente.

4. Retroalimentación: se presentó un alto grado de retroalimentación entre los estudiantes que estaban agrupados con un constante diálogo entre ellos sobre los aspectos que debían cubrir en las etapas iniciales del método, siendo constante durante la observación. Los estudiantes que trabajaron de manera 
individual, si bien dialogaban entre ellos, la retroalimentación se centró en la posibilidad de reiterar en algunos de los pasos que requería la recolección de información; además, se presentó una situación específica en estos estudiantes como fue el consultar a personas de su núcleo familiar sobre algunos aspectos del grupo objetivo que pedía la tarea.

5. Interacción: se observó que los estudiantes, no importando la agrupación o el trabajo individual, interactuaron con el objetivo de resolver algún tipo de preguntas; esto evidencia un alto nivel de razonamiento, por lo que el modelo logra concretar las acciones más relevantes del proceso de diseño.

6. Calidad de la tarea: esta categoría de observación permitió revisar la comprensión del modelo, la asimilación del ejercicio propuesto, la integración y cumplimiento de los pasos señalados en el modelo, eficacia en la realización del ejercicio y la claridad en la sustentación del mismo, estas acciones se manifiestan en la organización que los estudiantes demostraron en la presentación de las piezas gráficas.

Los resultados que arrojó el tercer cuestionario se basan en preguntas que los estudiantes resolvieron de manera amplia, posterior a la aplicación del modelo y la conclusión del ejercicio; entre sus respuestas y como ventajas del modelo DVUC-001 se destaca lo siguiente: permite organizar la información relacionada con el problema; incrementa la consciencia sobre el proceso convirtiéndolo en algo menos intuitivo; permite la integración diseñador cliente; favorece la retroalimentación y la interacción; mejora la estructuración del problema; segmenta el problema en partes; incluye pasos de verificación y brinda seguridad sobre la calidad del proceso.

Las desventajas identificadas por los estudiantes fueron: que el modelo DVUC001 exige demasiado tiempo para su aplicación; la reiteración en las fases 
que lo componen puede ser excesiva; el modelo no contempla un paso para realizar consultas con especialistas; no permite la exploración de soluciones alternas; el modelo cuenta con demasiados pasos, algunos de los cuales podrían fusionarse; el lenguaje utilizado para las instrucciones puede ser confuso, por ejemplo, los estudiantes no alcanzaron a percibir una diferencia significativa entre las fases visualización y jerarquización.

A la pregunta sobre la aplicación del modelo DVUC-001 a las diversas áreas que componen el Programa de Diseño Visual, los estudiantes manifestaron que era aplicable por su coherencia y por la generación de una imagen mental clara de lo que se debe realizar; condicionan su aplicación a una característica multidisciplinar y a la fusión de algunas de sus fases, de modo que se haga más concreto.

Finalmente a la identificación de los aspectos que ofrece el modelo DVUC001 para ser aplicado por los estudiantes, los participantes indicaron que la propuesta debe expandirse a proyectos diferentes a lo gráfico —ambiental, interfaces o audiovisual-; asimismo, el modelo ofrece: claridad en la instrucción, lo que permite la obtención de un resultado objetivo; posibilita la sustentación más coherente de los proyectos realizados; favorece la realización de un alto número de bocetos y, cuando se conforman grupos de trabajo, permite la definición y asignación de roles.

\section{Discusión y conclusiones}

En primer lugar, las desventajas que los participantes mencionaron permiten apreciar la valoración que tienen con los modelos de método, no exclusivamente con el propuesto; al valorar el modelo DVUC-001 las consideraciones que se hacen de su aplicabilidad, facilidad y demás aspectos relacionados con su validez se observa que alcanza cierto grado de equiparación con otros modelos 
utilizados debido a que posee una estructuración adecuada para su uso en los entornos para los que se proyectó. En ese mismo orden de ideas, es todavía común encontrar cierta reticencia a asumir al diseño desde una perspectiva menos intuitiva y creativa; entendida esta última como expresión de la libertad estética, que minimiza las exigencias que puedan desprenderse del contexto.

Ahora bien, la poca flexibilidad referida por los participantes sobre la estructura del modelo atiende a la coerción que un proceso metodológico requiere pero que al interior de la actividad proyectual puede resultar complejo de asimilar; pues la necesidad de prefigurar en la mente de los diseñadores una respuesta inmediata a los requerimientos verbales, es tal vez uno de los rezagos más fuertes de la formalización por intuición. Esto no implica una valoración sobre la utilidad de la intuición en el diseño, sin embargo, se hace necesario revisar la estructura del modelo para alejarla de la idea de 'lista' que los métodos en diseño poseen; y que como proceso, que requiere transformación, está sometido a la secuencialidad y contigüidad de la dimensión temporal.

Existe un aspecto que debe ser apreciado con mayor detenimiento, el modelo propuesto generó una serie de premisas que deben ser estructuradas para posteriores investigaciones entre ellas: al ser desarrollado a partir de teorías trabajadas en los contextos más conocidos por los participantes estos determinaron la utilidad del modelo y tuvieron cierta cercanía con el mismo, facilitando la comprensión de algunas de las etapas del proceso de diseño al reconocer acciones y logros expresados en estructuras teóricas manejadas con anterioridad. Esto no quiere decir que el proceso de diseño sea completamente diáfano para los participantes de estudio, no; por el contrario, sigue siendo muy dificultoso simplificar el proceso proyectual.

Otros aspectos Ilaman particularmente la atención de los investigadores en lo que respecta a las desventajas que se evidenciaron en su aplicación, la 
imposibilidad de realizar consultas con especialistas y lo restrictivo del modelo para la exploración de soluciones alternas. El primero de estos hace hincapié en la necesidad de los participantes de realizar consultas con especialistas, lo que denota una propensión del trabajo del diseñador a lo interdisciplinar; tesis que ya ha sido mencionada por otros autores, pero que en el trabajo específico con el modelo desarrollado no interfiere con la estructura del mismo puesto que a juicio de los investigadores es un factor exógeno a la aplicación del método debido a que se refiere más a la dinámica misma del ejercicio proyectual, Frascara (2006) lo enuncia de la siguiente manera: "el diseñador así coordina investigación, concepción y realización, haciendo uso de información o de especialistas de acuerdo con los requerimientos de los diferentes proyectos" (p. 25).

Sobre lo restrictivo del modelo para la exploración de soluciones alternas, los investigadores concluyen que los participantes identifican la prescripción del modelo para piezas gráficas como una limitación hacia otros soportes; es decir que el modelo se enfoca en obtener piezas gráficas, pero los diseñadores hoy deben considerar una serie de prototipos para diversos medios (audiovisuales, espaciales e informáticos), lo que refieren como una condición taxativa del modelo.

Es necesario mencionar que el modelo DVUC-001 permitió observar su equivalencia en la efectividad para realizar propuestas de tipo gráfico, ya que al ser un modelo de método tiene una aplicación tangible y que expresa con claridad el trasegar por el proceso de diseño; el modelo además recoge las teorías más ampliamente estudiadas durante el transcurso de la carrera y las sistematiza en las fases que lo componen, lo que permite inferir que muchos de los modelos metodológicos que se encuentran en el campo del diseño omiten su estructuración desde las teorías que los fundamentan; esto, en principio, puede dilatar el desarrollo mismo de la disciplina. 
Los estudiantes califican como muy importante contar con un modelo de método desarrollado al interior de su unidad académica que permita la realización de piezas de diseño, en este caso realizaciones de tipo gráfico, y no solo contar con métodos devenidos de las áreas del diseño industrial que pocas veces pueden ser adaptados completamente.

\section{Referencias}

Archer, B. (1968). Systemic methods for designers. London, England: Royal College of Art.

Arnheim, R. (1978). Pensamiento visual. Barcelona, España: Paidós.

Aumont, J. (1992). La imagen. Barcelona, España: Paidós.

Barthes, R. (2009). Lo obvio y lo obtuso. Barcelona, España: Paidós.

Bürdeck, B. (2002). Diseño. Historia, teoría y práctica del diseño industrial. Barcelona, España: Gustavo Gili.

Dussel, E. (1984). Filosofía de la producción. Bogotá, Colombia: Editorial Nueva América.

Fernández, S. y Bonsiepe, G. (2008). Historia del diseño en América Latina y el Caribe. São Paulo, Brasil: Editorial Blüncher.

Feyerabend, P. (1975). Contra el método. Barcelona, España: Ariel.

Frascara, J. (2006). El diseño de comunicación. Buenos Aires, Argentina: Editorial Infinito.

González Ruiz, G. (1994). Estudio de diseño. Buenos Aires, Argentina: Emecé Editores. 
Martí Font, J.M. (1999). Introducció a la metodologia del disseny. Barcelona, España: Universitat de Barcelona.

Moles, A. y Janiszewski, L. (1992). Grafismo funcional. Barcelona, España: CEAC.

Rodríguez, L. (1990). Para una teoría del diseño. Ciudad de México, México: Editorial Tilde.

Sanz, J.C. (1996). El libro de la imagen. Madrid, España: Alianza.

Villafañe, J. y Mínguez, N. (1996). Principios de teoría general de la imagen. Madrid, España: Editorial Pirámide.

Como citar: Ospina-Toro, W. (2019). Exposición del modelo DVUC-001 para la realización de productos gráficos en diseño visual. Revista KEPES, 16 (19), 151-182. DOI: 10.17151/kepes.2019.16.19.7 